\title{
Managing information overload
}

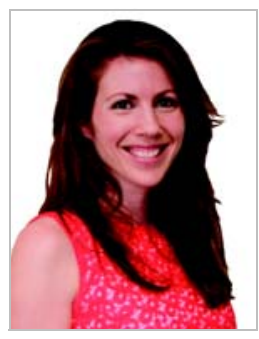

Christine Gee Deputy Editor mja@mja.com.au

doi: 10.5694/mjal4.c0421 (page 376) provides her perspective on how conflicting media reports of venous thromboembolism (VTE) risk have perplexed and caused undue worry for women choosing oral contraceptives. She recommends responding to this with an individualised discussion and risk analysis. She also suggests putting the VTE risk of the oral contraceptive pills in the context of other risk factors - including the more than 30-times higher risk of VTE postpartum.

Along this vein, Mazza and colleagues (page 414) have drawn data from the BEACH (Bettering the Evaluation and Care of Health) survey that suggest that since 2004, when emergency contraception was made available over the counter, women have had fewer consultations with their GPs for it. Yet the rates of consultations for unplanned pregnancies over the study period remained unchanged. The authors note a mismatch in the sense that women would prefer to receive information on emergency contraceptive options from their GP, but they are in fact obtaining this elsewhere.

In a cautionary note, Warner and colleagues (page 419) describe a case of internet shopping gone wrong. They report on a slimming supplement purchased from an unregulated online supplier. While this supplement was purported to be herbal, it unfortunately resulted in thyrotoxicosis and hepatitis due to pharmacologically active, hidden ingredients.

In contrast to the dangers described above, innovations in communication technology can be used to advantage in health care delivery. Leeb and colleagues (page 416) report an evaluation of a novel adverse event monitoring system for use after vaccination. They used SMS texts to follow up patients and carers, achieving a response rate of more than $70 \%$, and suggest that this has the potential to safely streamline surveillance activities.

Working hours is another topic featuring in this issue. Norman and Hall (page 399) have drawn data from the Medicine in Australia: Balancing Employment and Life (MABEL) study, showing that over $40 \%$ of surveyed GPs expressed a desire to reduce their total hours worked. Predictors of this included working more than 40 hours weekly and having on-call duties. In the subsequent year of the survey, only $26.8 \%$ of those reporting this preference actually managed to reduce their weekly working hours by 5 or more. This is not simply a matter of lifestyle convenience, as Kevat and colleagues (page 396) debate. They assert that there is strong evidence that long shifts and sleep deprivation lead to patient harm.

The use of nurse practitioners to reduce the workload on the medical workforce has been hotly debated. Bowyer and Schofield (page 382) explore the participation of this professional group in the context of oncology care. Specific tasks and special contributions under supervision may take pressure off doctors.

The central role of the doctor in providing accurate, humane information in the clinical setting needs support and recognition. This is part of the professional side of practice that grows in importance daily as the technology-based information cascade continues.

\section{Contracts and the brain drain}

cswannell@mja.com.au

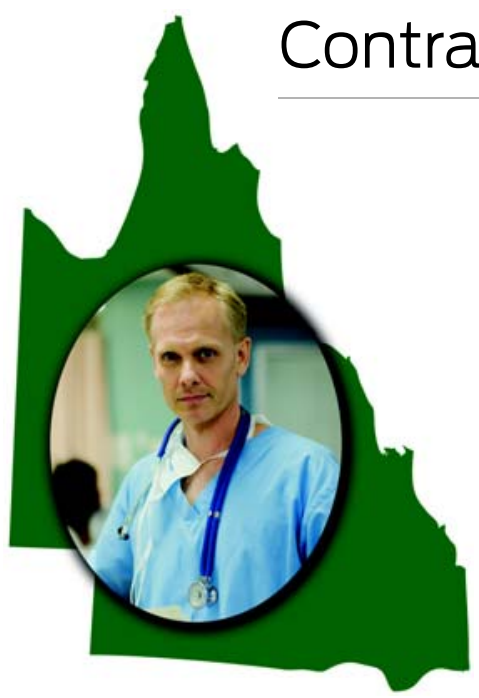

Mass industrial action has been a relatively rare occurrence in Australian medicine, but three decades on from the national visiting medical officers (VMOs) dispute, Queensland doctors are now mired in one of the worst industrial disputes the Australian medical profession has ever seen, with the potential to cripple Queensland's public hospital system. By the start of April at the Sunshine Coast's Nambour Hospital, the situation had driven a huge number of the hospital's specialist staff to say they would offer their resignations on the governmentimposed 30 April deadline if the contracts remained as they were. And there were similar stories coming out of hospitals from Cairns to Caboolture. How did it come to this? And what are the implications, if any, for VMOs and senior medical officers in the rest of the country (page C1)? The subject of our professional profile is Dr Graeme Duke, an intensivist who also spends time in the villages of Vanuatu, going back to the basics of primary care (page C3). 